\title{
La determinación del concepto de homogeneidad versus resistencia en los pilares de una catedral gótica mediante técnicas no invasivas
}

\author{
Determination of the homogeneity $v$ s. strength concept for columns of a \\ gothic cathedral by means of non-invasive techniques
}

J. Lluis i Ginovart $^{(*)}$, A. Costa-Jover ${ }^{(*)}$, S. Coll-Pla ${ }^{(*)}$

\section{RESUMEN}

La construcción y talla de los pilares en la construcción gótica son la base de la descarga de los empujes de las bóvedas hacia el terreno. La determinación morfológica y tipológica de estos elementos es esencial. En la catedral de Tortosa se experimenta un modelo estructural con doble deambulatorio, construyendo sus capillas entre 1377 y 1424. Éstas tienen la peculiaridad de no disponer de muro de separación entre ellas, como era frecuente en el gótico meridional. La eliminación del muro fue una cuestión debatida posteriormente en la catedral de Milán en 1392. El diseño y modelización de estos pilares, mediante técnicas no invasivas, utilizando el láser terrestre y el georadar, ha permitido constatar diferencias esenciales en su tipología. Se ha determinado que estos pilares son solamente homogéneos en los puntos de transmisión importantes de las cargas de la fábrica, que son en la basa y las tres primeras hiladas del fuste, así como en la parte superior de la imposta.

Palabras clave: escáner láser terrestre; georadar; construcción gótica; pilares; catedral Tortosa.

\section{ABSTRACT}

The construction and stone-cutting of columns in Gothic construction rules the transmission of the thrusts of the vaults to the ground. The morphologic and typological definition of these elements is essential. In the cathedral of Tortosa was defined an experimental structural model with a double ambulatory, with the construction of the ring of chapels between 1377 and 1424. These chapels are singular since they are not separated by a wall, as uses in Meridional Gothic. The suppression of this wall was after debated in the Cathedral of Milan in 1392. The survey of these columns through noninvasive techniques, such as Terrestrial Laser Scanner and Ground Penetrating Radar has enabled to identify essential differences between them. The study concluded that these columns are homogeneous only in the base and the first three rows of the body and near the impost, which are the areas were concentrates the main transmission of loads.

Keywords: terrestrial laser scanner; georadar; Gothic construction; columns; Tortosa Cathedral.

(*) Universidad Rovira y Virgili (Tarragona, España).

Persona de contacto/Corresponding author: arquitortosa@gmail.com (J. Lluis i Ginovart)

Cómo citar este artículo/Citation: Lluis i Ginovart, J., Costa-Jover, A., Coll-Pla, S. (2016). La determinación del concepto de homogeneidad versus resistencia en los pilares de una catedral gótica mediante técnicas no invasivas. Informes de la Construcción, 68(543): e155, doi: http://dx.doi.org/10.3989/ic.15.119.

Licencia / License: Salvo indicación contraria, todos los contenidos de la edición electrónica de Informes de la Construcción se distribuyen bajo una licencia de uso y distribución Creative Commons Attribution License (CC BY) Spain 3.o. 


\section{INTRODUCCIÓN}

La construcción gótica se basaba en unos criterios pre-científicos de lo que hoy se reconoce como obra de fábrica. La conocida como ponderibus, mecánica, y la de ingeniis, ingeniería, formaban parte de la matemática, según la De Scientiis, de Domingo de Gundisalvo (fl. 1150), y trasmitida a la Europa de las catedrales por Vicent de Beauvais (c. 1194-1264) en Speculum Doctrinale (1). Los constructores medievales asignaban diferentes conceptos al material y forma de la piedra, heredados de su saber práctico de la fabrica. Así la dureza de la piedra utilizada, se le asignaba el concepto de resistencia. A la talla de piedra y unión con morteros lo asignaríamos al de rigidez, y a la forma de los muros con sus pliegues y pilares al de estabilidad. Estos conceptos utilizados desde la praxis gótica pueden ser reconocidos en la moderna teoría elástica.

El objetivo del estudio es completar la modelización y conocimiento de los pilares de las capillas radiales del ábside de la catedral gótica de Tortosa (2), utilizando técnicas no destructivas, mediante una acción combinada de técnicas topográficas, escáner láser terrestre y georadar. Cada vez son más habituales los procedimientos topográficos y sobre todo de escáner láser en el patrimonio construido, y permiten realizar mediciones de objetos inaccesibles (3), (4). Así es posible capturar una gran cantidad de información sin entrar en contacto con la superficie del objeto a representar. Su fiabilidad ha sido ampliamente testeada (5), (6) y se complementa perfectamente con otras técnicas topográficas más tradicionales (7). Por otra parte, la prospección por medio de radar de im- pulsos, habitualmente utilizada en la localización de estructuras en el subsuelo (8), (9), se ha utilizado para determinar la composición interna de la estructura de fábrica, como en el caso de Santa María del Mar, en Barcelona (10), o la catedral de Santa María, de Mallorca (11).

Los pilares de las capillas radiales, construidos entre (13771424) alrededor la cabecera de la catedral románica, construyen un doble deambulatorio con una sección muy baja de proporción 9/5, lo que supuso, por primera vez, la supresión del muro de las capillas del ábside (Figura 1). Este problema estructural será planteado años más tarde en la construcción de la catedral de Milán de 1392 (12). Posteriormente, en la construcción del deambulatorio (1432-1434) hubo un cambio de sección, pasando a una proporción más alta de 9/6, que también se da en el presbiterio (1439-1440). El elemento esencial para la construcción de este modelo estructural es el pilar de las capillas radiales, puesto que ha de recoger los empujes iniciales de estas capillas, así como los futuros del segundo nivel del deambulatorio. El diseño del pilar de Tortosa es semejante a los salmeres de la tradición francesa, así se construyen mediante elementos diferenciados que se encajan en el enjarje del pilar (13). Esta tipología permite la descarga de los arcos torales, formeros y cruceros de las bóvedas concurrentes, tanto en su intradós, hacia el deambulatorio, como las del extradós hacia las capillas.

\section{MATERIAL Y METODOLOGÍA}

El objetivo es determinar las características constructivas de los 8 pilares $\left(\mathrm{P}_{2} \ldots \mathrm{P}_{9}\right)$ y las dos pilastras $\left(\mathrm{P}_{1}, \mathrm{P}_{10}\right)$, los cua-
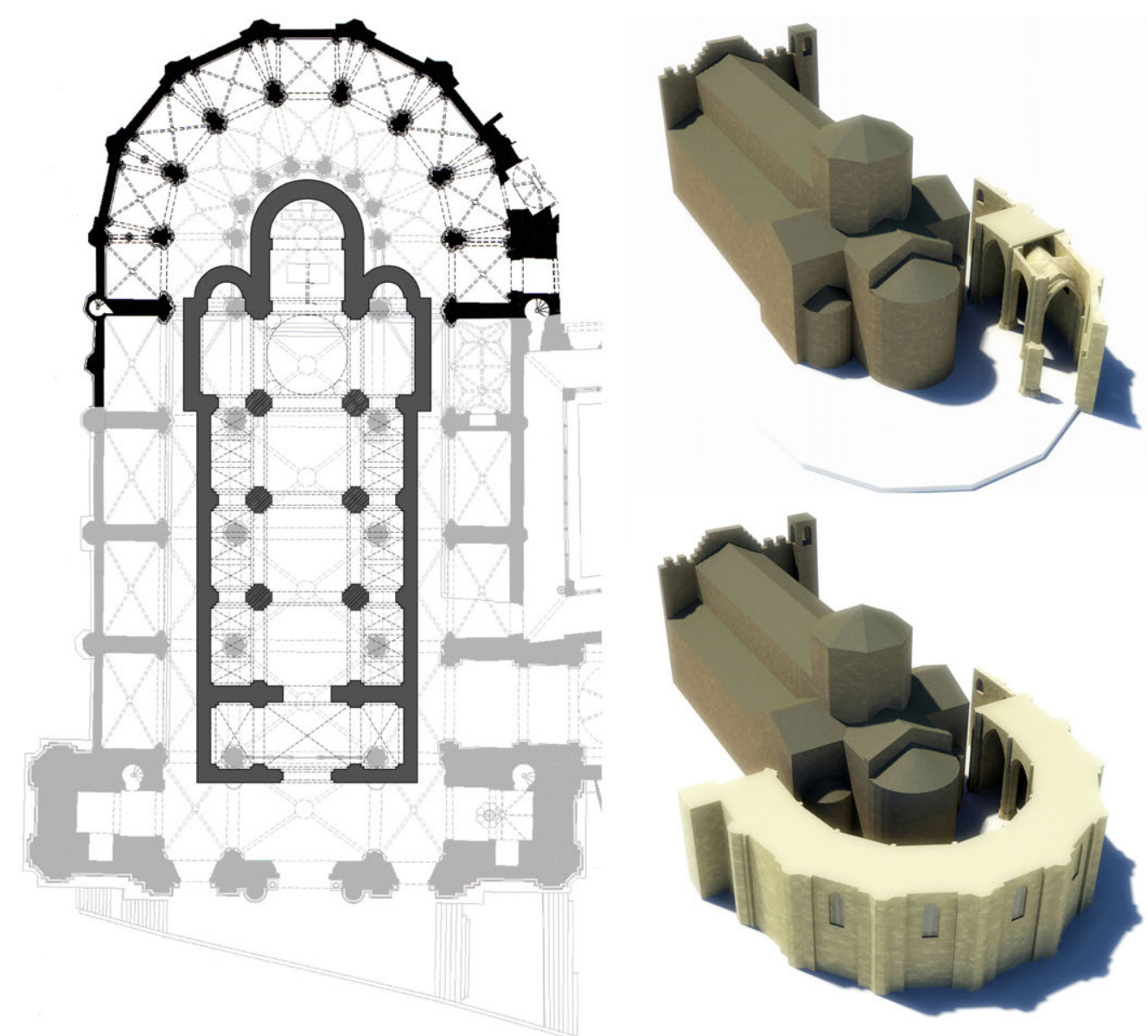

Figura 1. Construcción de las capillas radiales de la nueva cabecera gótica, rodeando la catedral románica (1377-1424). 
les sustentan las bóvedas de las 9 capillas radiales $\left(\mathrm{C}_{1} \ldots \mathrm{C}_{0}\right)$, por medio de técnicas no invasivas de georadar y topográficas (Figura 2). Así se establecerá la geometría precisa de los pilares, su despiece y su composición interior, incluyendo el relleno de los senos del arranque de las bóvedas. Con ello se pretende una aproximación a la epistemología de la construcción del gótico meridional, desarrollando una metodología de reconocimiento del patrimonio.

\subsection{Determinación geométrica}

La operativa para la determinación geométrica combina los datos topográficos obtenidos a partir de un escáner láser terrestre (TLS), modelo Leica ScanStation $\mathrm{C}^{1} \mathrm{O}^{1}$, que captura la geometría general de los elementos constructivos, y los de una estación total Topcon ${ }^{2}$, con la que se establece la altura de las hiladas en cada uno de los pilares. El procesado de datos (TLS) se realiza con los programas «Cyclone», para la composición de nubes de puntos, y «3D Reshaper» para el mallado, obteniendo una malla TIN (Triangulated Irregular Network) con un lado promedio de $0,033 \mathrm{~m}$, y una detección de orificios en la malla determinada por un tamaño de triángulo de 0,099 m. Los datos del escáner permiten definir la geometría general de los pilares, pero no su despiece, debido al escaso relieve entre las juntas. Para ello se realiza el topografiado convencional de las hiladas, mediante dos posicionamientos, referenciados a través de puntos comunes identificados en el relieve de la fábrica. Se toma como referencia para las mediciones la imposta del arco toral situado en la girola. Complementariamente, se cuantifica el número de piezas que conforma cada hilada, proceso que se realiza in situ por medios organolépticos. Con ello es posible determinar diferencias geométricas de los pilares, así como el despiece de la mampostería de los 8 pilares $\left(\mathrm{P}_{2} \ldots\right.$ $\left.\mathrm{P}_{9}\right)$ y las dos pilastras $\left(\mathrm{P}_{1}, \mathrm{P}_{10}\right)$, analizando si hay diferencias significativas en el período de ejecución (1377-1424).

\subsection{Composición interna}

La determinación de la calidad del interior del pilar se realiza mediante un georadar (GPR) IDS Fast-Wave, con una antena de alta frecuencia de $900 \mathrm{MHz}^{3}$. Se desarrolló una exploración sistemática de los elementos $\left(\mathrm{P}_{1} \ldots \mathrm{P}_{10}\right)$ mediante perfiles simples de sección vertical longitudinal y transversal.

Los datos del georadar se procesaron siguiendo una secuencia que incluye:

[a] reconocimiento de la superficie de entrada de la señal;

[b] corrección de una deriva especifica del sistema;

[c] compensación de la atenuación de la señal con la profundidad;

[d] conversión del tiempo de propagación de la señal a profundidad;

Los datos recogidos por el georadar son de carácter bipolar y corresponden a las reflexiones de un pulso de energía que

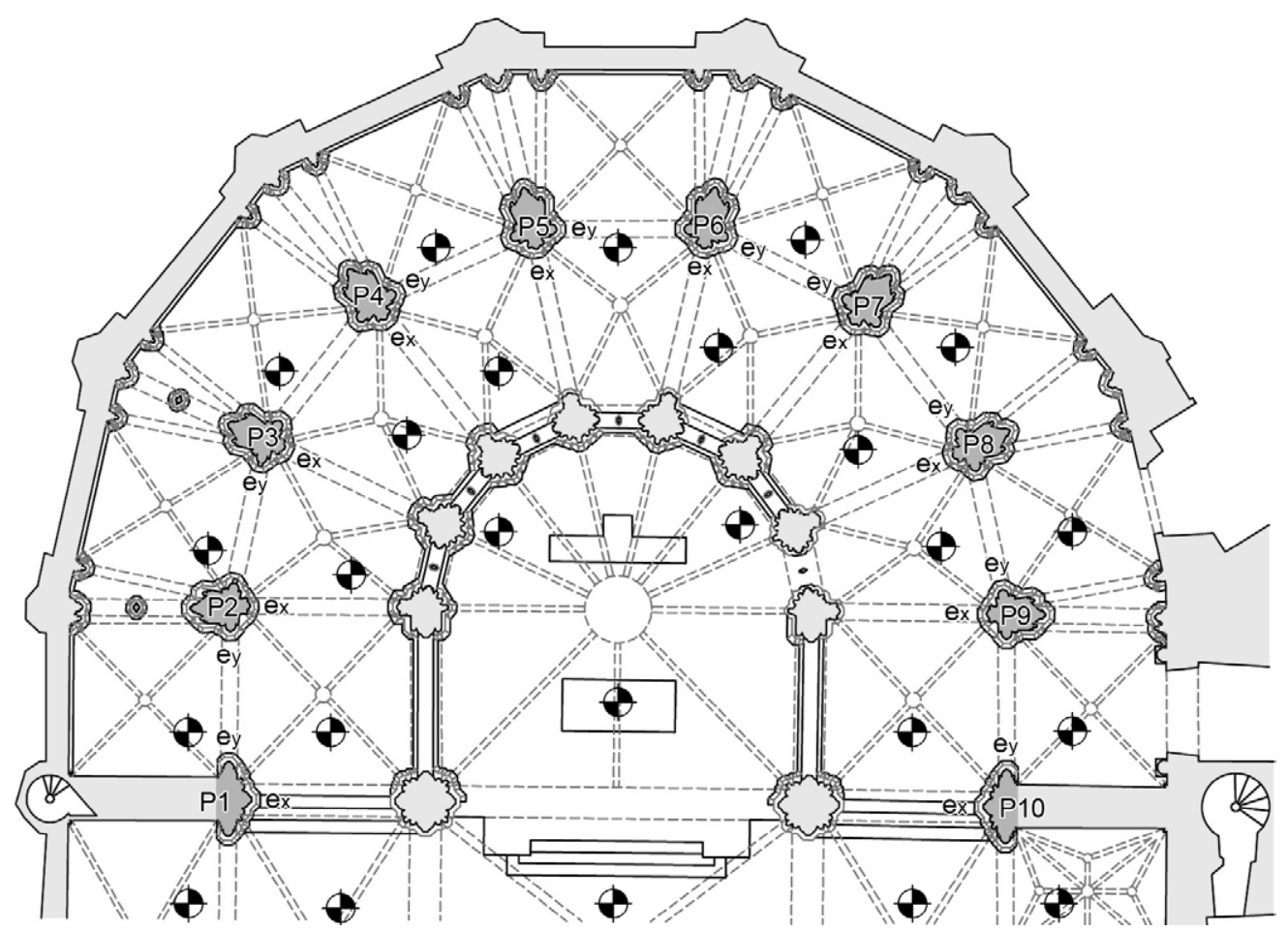

Figura 2. Posicionamientos del escáner láser, nomenclatura y secciones de georadar.

\footnotetext{
${ }^{1}$ Campaña Lluis, Costa, Coll, (2014-2015), con 25 estaciones, con escáner láser de muy alta velocidad 50.0oo puntos/seg. Modelo con doble eje, plomada láser, clase de láser: 2 (CEI 60825-1). Óptica con un espejo Smart X-Mirror ${ }^{\mathrm{TM}}$. El láser 3R (IEC 60825-1), con longitud de onda $532 \mathrm{~nm}$ visible. Rango de precisión; posición ( $6 \mathrm{~mm}$ ), distancia (4 mm), ángulo horizontal/vertical (6o $\mu \mathrm{rad} / 60 \mu \mathrm{rad}$; 12 "/12”).

${ }^{2}$ Topcon Imaging Station 203, de precisión de 0,2 mm/1 mm \pm (5 mm). Cámaras (2) 1,3 mp, velocidad de imagen 1-10 fps, exploración máx. $20 \mathrm{pts} / \mathrm{seg}$.

${ }_{3}^{3}$ Sala; Tamba (2015). SOT Prospecció Arqueològica. Resolución vertical o,01 m. Resolución en profundidad o,005 m. Número de muestras por traza 512, ventana con tiempo de adquisición de $45 \mathrm{~ns}$. Velocidad de propagación estimada $0,122 \mathrm{~m} / \mathrm{ns}$ calculada tomando un perfil de calibración del muro lateral del pilar p. 10 de grueso conocido.
} 
oscila a través de los medios de propagación. Las representaciones de los datos responden a diferentes formas de expresar una misma información. Inicialmente se establecen dos modalidades de visualización para poder describir las anomalías internas detectadas. Éstas expresan las variaciones relativas de los datos obtenidos. Complementariamente, se propone un proceso alternativo que incluye dos pasos adicionales (Figura 3):

[b2] un filtro background que extrae la onda media de los datos;

[e] la migración de los datos que permite focalizar las reflexiones de los elementos de la geometría compleja.

La primera modalidad es una visualización en amplitud de señal reflejada. Corresponde a las variaciones de fase de la señal que se amplifica cuando se encuentra en una interface entre objetos de materiales diferentes. Cada interface está asociada a un polo negativo y a otro positivo.

La segunda es una visualización en magnitud de señal reflejada [1]. Corresponde a la media del valor absoluto de los valores de amplitud calculada en una ventana móvil de $8,6 \mathrm{~cm}$, que se establece a partir de los datos obtenidos:

$$
\text { Magnitud }=\sum_{i}^{n} \mid \text { amplitud } \mid
$$

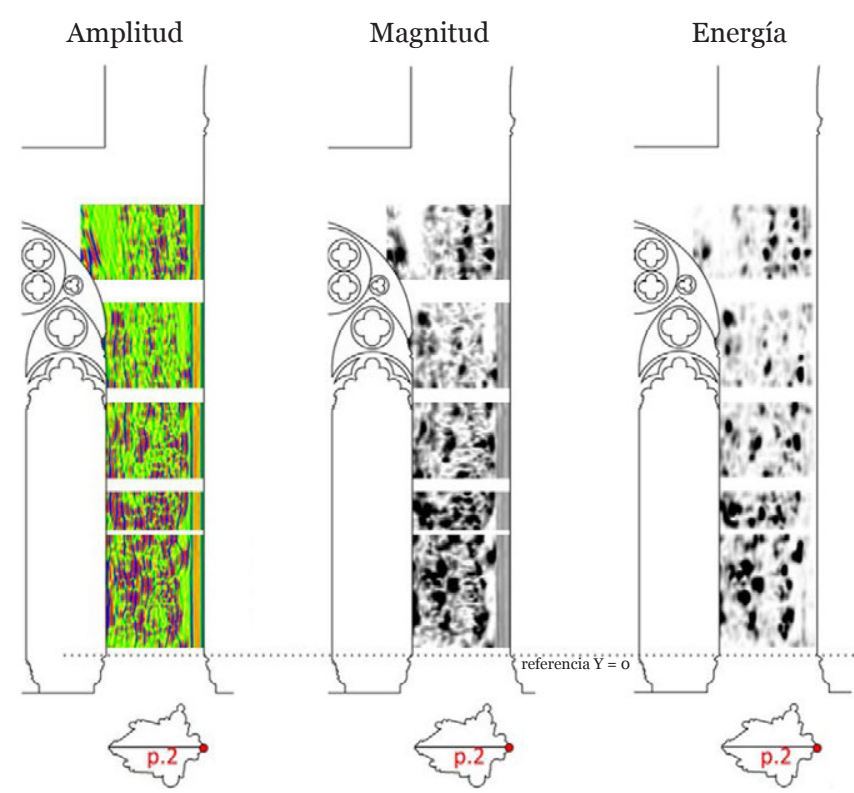

Figura 3. Visualizaciones de los resultados del georadar. donde $n$ es el número de muestras incluidas en la ventana móvil. El proceso permite definir la geometría de los elementos detectados y diferenciar las partes homogéneas de les partes alteradas.

El proceso alternativo permite observar diferencias de menor amplitud presentes en las partes homogéneas [2]. Los datos con este proceso se visualizan en una tercera modalidad, la de la energía de señal reflejada. Corresponde a la media del valor al cuadrado del valor de amplitud calculada en una ventana móvil también de $8,6 \mathrm{~cm}$, permitiendo así aumentar el contraste de los elementos detectados;

$$
\text { Energía }=\sum_{i}^{n} \text { amplitud }^{2}
$$

\section{RESULTADOS}

\subsection{Escáner láser terrestre}

Los resultados del análisis topográfico permiten incidir en la morfología y el despiece vertical de los pilares.

1. Morfológicamente existe una diferencia formal entre las dos pilastras extremas $\left(\mathrm{P}_{1}, \mathrm{P}_{10}\right)$ construidas sobre el muro que cierra el ábside, y los ocho pilares del doble deambulatorio $\left(\mathrm{P}_{2} \ldots \mathrm{P}_{9}\right)$. También existen variaciones entre las medidas de los pilares, de manera que los pilares $\left(\mathrm{P}_{2}, \mathrm{P}_{9}\right)$ no son simétricos sobre su sección principal $e_{x}$, mientras que los seis pilares restantes $\left(\mathrm{P}_{3} \ldots \mathrm{P}_{8}\right)$ presentan simetría sobre su eje mayor (Figura 4.1). La diferencia entre estas dos familias de pilares se acentúa también en las dimensiones de su sección principal $e_{x}$, así como en los dos ejes transversales $e_{y 1}$ y $e_{y 2}$, con la correspondiente repercusión en la diferente sección de $\left(s_{1}, s_{9}\right)\left(s_{3} \ldots s_{8}\right)$ (Tabla 1$)$. Existen diferencias geométricas importantes en la sección vertical de los pilares, de manera que se observan tres tipos de molduras diferentes en la base. La pilastra $\mathrm{P}_{1}$, la primera en ser construida, dispone de una moldura $\mathrm{M}_{\mathrm{A}}$, que se prolonga por el muro de cerramiento con sus pliegues hasta la posición del pilar $\mathrm{P}_{4}$. El pilar $\mathrm{P}_{2}$ dispone de una moldura $\mathrm{M}_{\mathrm{B}}$, mientras que los pilares $\left(\mathrm{P}_{3} \ldots \mathrm{P}_{9}\right)$ y la pilastra $\mathrm{P}_{10}$ disponen de una moldura tipo $\mathrm{M}_{\mathrm{C}^{\circ}}$ (Figura 4.2).

2. En el análisis de la sección vertical y el despiece de hiladas de los pilares y pilastras se observan diferencias importantes, tanto en la medida como en el número de piezas. Las medidas se realizan desde la cota cero de la base hasta la cara superior de la imposta del arco del deambulatorio (Tabla 2). La altura total oscila $0,190 \mathrm{~m}\left(\mathrm{P}_{2}=7,053 \mathrm{~m}-\right.$ $\left.\mathrm{P}_{10}=6,862 \mathrm{~m}\right)$. Si no se consideran las pilastras, este valor disminuye hasta los $0,133 \mathrm{~cm}\left(\mathrm{P}_{2}\right.$ a $\left.\mathrm{P}_{9}=6,920 \mathrm{~m}\right)$. El número de hiladas varía según el pilar en un rango entre 20

Tabla 1. Dimensiones de los pilares de las capillas radiales.

\begin{tabular}{|l|c|c|c|c|c|c|c|c|c|c|}
\hline \multicolumn{1}{|c|}{ Pilares } & P1 & P2 & P3 & P4 & P5 & P6 & P7 & P8 & P9 & P10 \\
\hline $\mathrm{Fe}_{x} / \mathrm{m}$ & 1,049 & 1,923 & 1,992 & 1,980 & 2,004 & 2,002 & 2,010 & 2,002 & 1,896 & 1,046 \\
\hline $\mathrm{Fe}_{y 1} / \mathrm{m}$ & 2,387 & 0,797 & 0,861 & 0,767 & 0,765 & 0,748 & 0,788 & 0,738 & 0,806 & 2,362 \\
\hline $\mathrm{Fe}_{y 2} / \mathrm{m}$ & & 0,903 & 0,780 & 0,769 & 0,783 & 0,732 & 0,760 & 0,763 & 0,795 & \\
\hline $\mathrm{Fs} . / \mathrm{m}^{2}$ & 1,611 & 1,677 & 1,755 & 1,742 & 1,769 & 1,684 & 1,787 & 1,709 & 1,681 & 1,593 \\
\hline $\mathrm{Bex} / \mathrm{m}$ & 1,375 & 2,456 & 2,551 & 2,540 & 2,564 & 2,562 & 2,570 & 2,562 & 2,456 & 1,375 \\
\hline $\mathrm{Be}_{y 1} / \mathrm{m}$ & 1,464 & 1,079 & 1,029 & 1,044 & 1,048 & 1,012 & 1,040 & 1,043 & 1,075 & 1,458 \\
\hline $\mathrm{Be}_{y 2} / \mathrm{m}$ & 1,483 & 1,086 & 1,063 & 1,052 & 1,060 & 1,027 & 1,069 & 1,018 & 1,087 & 1,461 \\
\hline $\mathrm{Bs} . / \mathrm{m}^{2}$ & 3,013 & 3,695 & 3,726 & 3,741 & 3,782 & 3,668 & 3,807 & 3,689 & 3,695 & 2,984 \\
\hline $\mathrm{Moldura}$ & $\mathrm{Ma}$ & $\mathrm{Mb}$ & $\mathrm{Mc}$ & $\mathrm{Mc}$ & $\mathrm{Mc}$ & $\mathrm{Mc}$ & $\mathrm{Mc}$ & $\mathrm{Mc}$ & $\mathrm{Mc}$ & $\mathrm{Mc}$ \\
\hline
\end{tabular}


Tabla 2. Despiece $(n)$ y alturas $(h)$ de las hiladas de los pilares y las pilastras.

\begin{tabular}{|c|c|c|c|c|c|c|c|c|c|c|c|c|c|c|c|c|c|c|c|c|c|c|c|c|c|c|}
\hline & \multicolumn{2}{|l|}{ B1 } & \multicolumn{2}{|l|}{ B2 } & \multicolumn{2}{|l|}{ H1 } & \multicolumn{2}{|l|}{ H2 } & \multicolumn{2}{|l|}{ H3 } & \multicolumn{2}{|l|}{$\mathrm{H}_{4}$} & \multicolumn{2}{|l|}{ H5 } & \multicolumn{2}{|l|}{ H6 } & \multicolumn{2}{|l|}{$\mathbf{H}_{7}$} & \multicolumn{2}{|l|}{ H8 } & \multicolumn{2}{|l|}{ H9 } & \multicolumn{2}{|l|}{ H10 } & \multicolumn{2}{|l|}{ H11 } \\
\hline & $\boldsymbol{h}$ & $n$ & $\boldsymbol{h}$ & $n$ & $\boldsymbol{h}$ & $n$ & $\boldsymbol{h}$ & $n$ & $\boldsymbol{h}$ & $n$ & h & $n$ & \begin{tabular}{ll|}
$\boldsymbol{h}$ \\
\end{tabular} & $n$ & $\boldsymbol{h}$ & $n$ & $\boldsymbol{h}$ & $n$ & $\boldsymbol{h}$ & $n$ & $\boldsymbol{h}$ & $n$ & \begin{tabular}{l|l}
$h$ \\
\end{tabular} & $n$ & $\boldsymbol{h}$ & $n$ \\
\hline $\mathrm{P}_{1}$ & 0,343 & 5 & ,486 & 2 & 0,371 & 2 & 0,278 & 2 & 0,349 & 2 & 0,318 & 3 & 0,295 & 2 & 0,291 & 5 & 0,211 & 2 & 0,277 & 3 & 0,276 & 2 & 0,329 & 4 & 0,296 & 2 \\
\hline $\mathrm{P}_{2}$ & 0,343 & 2 & 0,443 & 6 & 0,322 & 4 & 0,345 & 4 & 0,316 & 5 & 0,367 & 4 & 0,226 & 5 & 0,297 & 6 & 0,347 & 6 & 0,259 & 5 & 0,383 & 6 & 0,241 & 5 & 0,351 & 6 \\
\hline $\mathrm{P}_{3}$ & 0,356 & 7 & 0,422 & 8 & 0,383 & 6 & 0,288 & 5 & 0,287 & 7 & 0,333 & 5 & 0,289 & 6 & 0,291 & 7 & 0,256 & 8 & 0,349 & 5 & 0,278 & 6 & 0,361 & 6 & 0,314 & 6 \\
\hline $\mathrm{P}_{4}$ & 0,339 & 5 & 047 & 6 & 0,336 & 6 & 0,330 & 7 & 0,257 & 6 & 0,291 & 6 & 0,294 & 6 & 0,224 & 5 & 0,255 & 6 & 0,275 & 4 & 0,238 & 8 & 0,265 & 5 & 0,265 & 7 \\
\hline $\mathrm{P}_{5}$ & 0,346 & 12 & 0,433 & 11 & 0,273 & 5 & 0,291 & 4 & 0,287 & 6 & 0,294 & 5 & 0,291 & 6 & 0,259 & 6 & 0,287 & 6 & 0,324 & 6 & 0,324 & 6 & 0,331 & 6 & 0,288 & 6 \\
\hline $\mathrm{P}_{6}$ & 0,257 & 12 & 0,496 & 11 & 0,312 & 6 & 0,300 & 4 & 0,250 & 6 & 0,322 & 7 & 0,262 & 6 & 0,177 & 6 & 0,308 & 6 & 0,295 & 6 & 0,228 & 7 & 0,287 & 6 & 0,292 & 6 \\
\hline$P_{7}$ & 0,339 & 6 & 0,428 & 6 & 0,288 & 7 & 0,252 & 6 & 0,260 & 6 & 0,221 & 4 & 0,247 & 5 & 0,257 & 4 & 0,287 & 5 & 0,285 & 5 & 0,290 & 5 & 0,261 & 5 & 0,286 & 5 \\
\hline $\mathrm{P}_{8}$ & 0,348 & 7 & 0,414 & 6 & 0,312 & 6 & 0,250 & 4 & 0,227 & 5 & 0,334 & 7 & 0,287 & 5 & 0,263 & 6 & 0,245 & 4 & 0,255 & 5 & 0,316 & 5 & 0,267 & 5 & 0,262 & 4 \\
\hline $\mathrm{P}_{9}$ & 0,329 & 4 & 0,434 & 4 & 0,284 & 7 & 0,286 & 3 & 0,290 & 6 & 0,244 & 4 & 0,251 & 4 & 0,264 & 5 & 0,225 & 5 & 0,296 & 5 & 0,246 & 5 & 0,231 & 4 & 0,283 & 3 \\
\hline$P_{10}$ & 0,324 & 5 & 0,428 & 4 & 0,314 & 2 & 0,317 & 2 & 0,326 & 2 & 0,328 & 2 & 0,196 & 3 & 0,340 & 2 & 0,264 & 2 & 0,294 & 2 & 0,228 & 2 & 0,261 & 4 & 0,244 & 3 \\
\hline
\end{tabular}

\begin{tabular}{|c|c|c|c|c|c|c|c|c|c|c|c|c|c|c|c|c|c|c|c|c|c|c|c|c|}
\hline & \multicolumn{2}{|c|}{ H12 } & \multicolumn{2}{|l|}{ H13 } & \multicolumn{2}{|c|}{ H14 } & \multicolumn{2}{|c|}{ H15 } & \multicolumn{2}{|c|}{ H16 } & \multicolumn{2}{|c|}{ H17 } & \multicolumn{2}{|l|}{ H18 } & \multicolumn{2}{|c|}{ H19 } & \multicolumn{2}{|c|}{ H2O } & \multicolumn{2}{|l|}{ H21 } & \multicolumn{2}{|c|}{ H22 } & \multicolumn{2}{|l|}{ H23 } \\
\hline & \begin{tabular}{ll|}
$h$ \\
\end{tabular} & $n$ & $\begin{array}{ll}\boldsymbol{h} \\
\end{array}$ & $\boldsymbol{n}$ & \begin{tabular}{l|l|}
$h$ \\
\end{tabular} & $n$ & $\begin{array}{ll}\boldsymbol{h} \\
\end{array}$ & $n$ & $\boldsymbol{h}$ & $n$ & h & $n$ & \begin{tabular}{l|l}
$\boldsymbol{h}$ \\
\end{tabular} & $n$ & $\boldsymbol{h}$ & $n$ & $\boldsymbol{h}$ & $n$ & \begin{tabular}{ll|}
$\boldsymbol{h}$ \\
\end{tabular} & $n$ & $\boldsymbol{h}$ & $n$ & h & $n$ \\
\hline $\mathrm{P}_{1}$ & 0,319 & 2 & 0,225 & 5 & 0,344 & 2 & 0,417 & $2 \mid$ & 0,343 & 2 & 0,206 & 2 & 0,275 & \begin{tabular}{|l|}
3 \\
\end{tabular} & 0,284 & 2 & 0,165 & 2 & 0,348 & 2 & & & & \\
\hline $\mathrm{P}_{2}$ & 0,318 & 3 & 0,307 & 3 & 0,207 & 5 & 0,336 & 5 & 0,441 & 4 & 0,279 & 4 & 0,248 & 2 & 0,294 & 2 & 0,381 & 2 & & & & & & \\
\hline $\mathrm{P}_{3}$ & 0,300 & 6 & 0,279 & 5 & 0,289 & 6 & 0,297 & 4 & 0,428 & 5 & 0,299 & 5 & 0,255 & 8 & 0,301 & 4 & 0,282 & 4 & & & & & & \\
\hline $\mathrm{P}_{4}$ & 0,337 & 5 & 0,203 & 6 & 0,260 & 6 & 0,257 & 5 & 0,290 & 5 & 0,227 & 6 & 0,231 & 6 & 0,262 & 5 & 0,284 & 5 & 0,261 & 4 & 0,295 & 3 & 0,281 & 3 \\
\hline $\mathrm{P}_{5}$ & 0,319 & 6 & 0,361 & 6 & 0,298 & 7 & 0,273 & 6 & 0,271 & 7 & 0,329 & 6 & 0,289 & 6 & 0,322 & 6 & 0,223 & 6 & 0,303 & 4 & & & & \\
\hline $\mathrm{P}_{6}$ & 0,224 & 6 & 0,260 & 6 & 0,256 & 6 & 0,256 & 6 & 0,292 & 6 & 0,229 & 6 & 0,225 & 6 & 0,322 & 8 & 0,286 & 6 & 0,256 & 7 & 0,292 & 6 & 0,289 & 7 \\
\hline $\mathrm{P}_{7}$ & 0,223 & 6 & 0,294 & 5 & 248 & 3 & 0,336 & 6 & 0,229 & 5 & 0,243 & 4 & 0,302 & 5 & 0,283 & 6 & 0,287 & 5 & 0,268 & 5 & 0,273 & 3 & 0,287 & 6 \\
\hline $\mathrm{P}_{8}$ & 0,223 & 4 & 0,322 & 4 & 0,283 & 4 & 0,256 & 5 & 0,227 & 3 & 0,289 & 5 & 0,257 & 2 & 0,283 & 5 & 0,287 & 2 & 0,266 & 6 & 0,285 & 4 & 0,286 & 4 \\
\hline $\mathrm{P}_{9}$ & 0,256 & 4 & 0,287 & 4 & 0,250 & 4 & 0,234 & 5 & 0,247 & 4 & 0,252 & 5 & 0,217 & 5 & 0,291 & 4 & 0,278 & 3 & 0,300 & 4 & 0,357 & 5 & 0,289 & 2 \\
\hline$P_{10}$ & 0,261 & 2 & 0,254 & 2 & 0,296 & 2 & 0,258 & 2 & 0,266 & 2 & 0,255 & 2 & 0,278 & 2 & 0,269 & 2 & 0,270 & 2 & 0,302 & 2 & 0,288 & 2 & & \\
\hline
\end{tabular}

Tabla 3. Número de piezas y alturas de los pilares.

\begin{tabular}{|l|c|c|c|c|c|c|}
\hline & Altura base & $\boldsymbol{n}$ base & Altura fuste & $\boldsymbol{n}$ fuste & Altura total & $\boldsymbol{n}$ total \\
\hline $\mathrm{P}_{1}$ & 0,829 & 7 & 6,215 & 51 & 7,044 & 58 \\
\hline $\mathrm{P}_{2}$ & 0,786 & 8 & 6,267 & 86 & 7,053 & 94 \\
\hline $\mathrm{P}_{3}$ & 0,778 & 15 & 6,158 & 114 & 6,936 & 129 \\
\hline $\mathrm{P}_{4}$ & 0,776 & 11 & 6,217 & 125 & 6,993 & 136 \\
\hline $\mathrm{P}_{5}$ & 0,779 & 23 & 6,240 & 122 & 7,019 & 145 \\
\hline $\mathrm{P}_{6}$ & 0,753 & 23 & 6,219 & 142 & 6,972 & 165 \\
\hline $\mathrm{P}_{7}$ & 0,767 & 12 & 6,207 & 116 & 6,974 & 128 \\
\hline $\mathrm{P}_{8}$ & 0,762 & 13 & 6,281 & 104 & 7,043 & 117 \\
\hline $\mathrm{P}_{9}$ & 0,763 & 8 & 6,157 & 100 & 6,920 & 108 \\
\hline $\mathrm{P}_{10}$ & 0,752 & 9 & 6,111 & 48 & 6,863 & 57 \\
\hline
\end{tabular}

(caso de los pilares $\mathrm{P}_{2}$ y $\mathrm{P}_{3}$ ) y 23 hiladas (pilares $\mathrm{P}_{4}$ y $\mathrm{P}_{6}$ a $\mathrm{P}_{9}$ ). La altura media de las hiladas se establece en $0,285 \mathrm{~m}$, con una dispersión entre las medias en cada pilar de 0,055 $\mathrm{cm}\left(\mathrm{P}_{2}=0,320 \mathrm{~m}-\mathrm{P}_{4}=0,265 \mathrm{~cm}\right)$. La dispersión entre las alturas máximas de hiladas se establece en $0,082 \mathrm{~m}\left(\mathrm{P}_{6}=\right.$ $\left.0,496 \mathrm{~m}-\mathrm{P}_{8}=0,414\right)$, y la dispersión mínima en $0,090 \mathrm{~m}$ $\left(\mathrm{P}_{3}=0,255 \mathrm{~m}-\mathrm{P}_{1}=0,165 \mathrm{~cm}\right)$.

3. Encontramos diferencias también significativas en el número de piezas por hilada. El número de piezas medio entre $\left(\mathrm{P}_{1}-\mathrm{P}_{10}\right)$ es de 122,5, siendo 165 el número máximo $\left(\mathrm{P}_{6}\right)$ y 57 el número mínimo $\left(\mathrm{P}_{10}\right)$, con una dispersión de 108 piezas. Si no se consideran las pilastras, la dispersión entre el número de piezas disminuye a 71 , siendo $\left(\mathrm{P}_{2}\right)$ el que tiene un número menor con 94 piezas. Los pilares $\left(\mathrm{P}_{4}\right.$, $\mathrm{P}_{5}, \mathrm{P}_{6}$ ), situados en la parte central del ábside, son los que tienen un número mayor de piezas en el fuste con 125, 122 y 142, respectivamente (Tabla 3).
En $\left(\mathrm{P}_{4}, \mathrm{P}_{5}, \mathrm{P}_{6}\right)$ se observa la aparición de un despiece diferente a $\left(\stackrel{4}{\mathrm{P}}_{2}, 5_{3}\right)$ con piezas más pequeñas, en el lado de los arcos perpiaños de descarga del ábside. En estas piezas aparecen marcas de sanguina con numeración romana. La numeración está situada al lado de las juntas, disponiendo de dos marcas (Tabla 4). La numeración es igual que la existente en los contrafuertes de la catedral de Colonia (14) (Figura 4.3).

\subsection{Resultados georradar}

Los resultados obtenidos de las secciones longitudinales $e_{x} \mathrm{y}$ transversales $e_{y}$ (Figura 5) revelan una composición interna de los pilares heterogénea. La propagación de las ondas electromagnéticas emitidas por el sistema de georadar tiene geometría cónica. La huella de propagación es específica de la antena utilizada, y su visibilidad aumenta con la profundidad. La interpretación de los resultados 

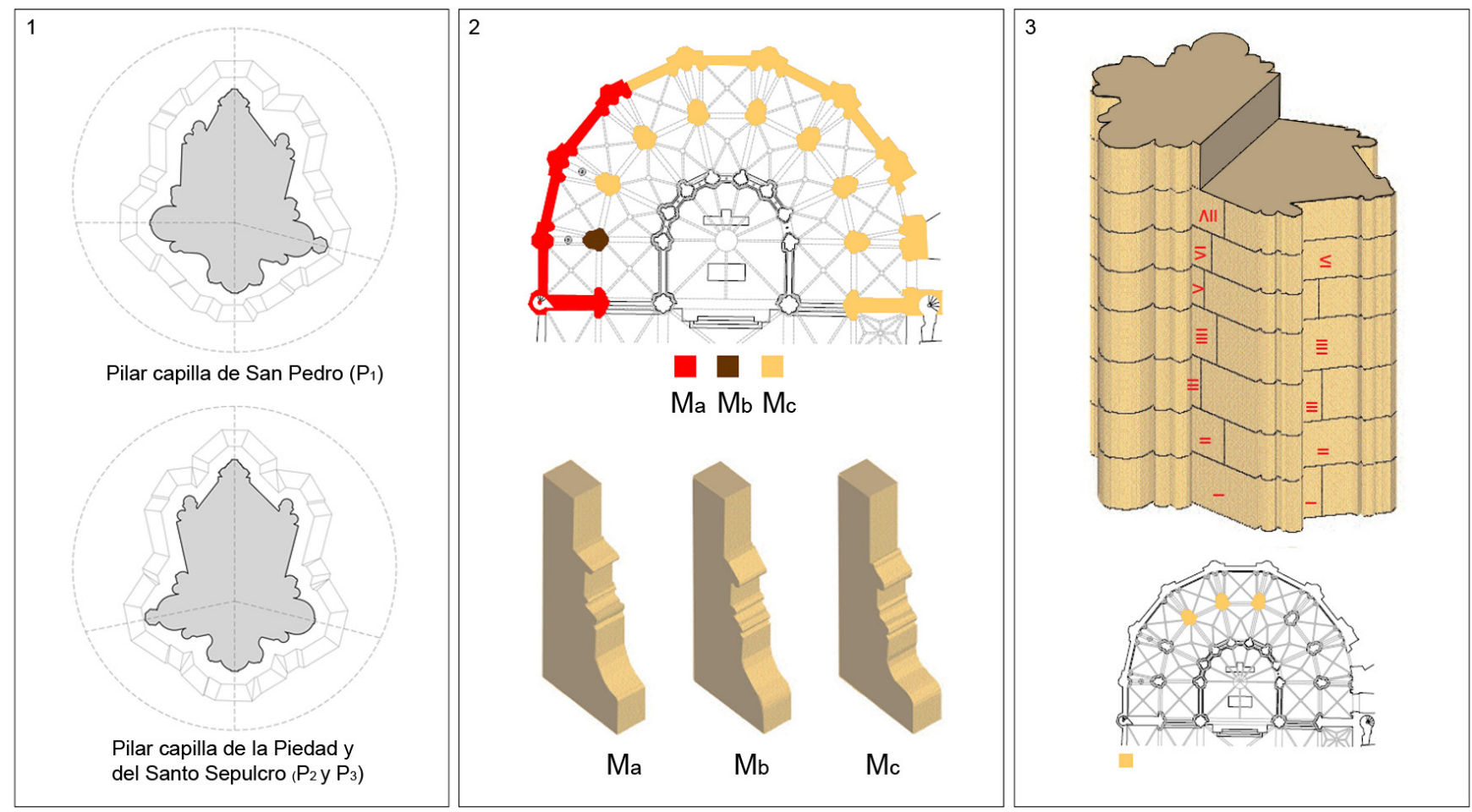

Figura 4. Características de los pilares: 1. diferencias morfológicas $\left(\mathrm{P}_{1}, \mathrm{P}_{2}, \mathrm{P}_{3}\right)$; 2. diferencias en las molduras; 3. numeración romana en los pilares $\left(\mathrm{P}_{4}, \mathrm{P}_{5}, \mathrm{P}_{6}\right)$.

Tabla 4. Numeración romana en el despiece de los pilares.

\begin{tabular}{|l|c|c|c|c|c|c|c|c|c|c|c|c|c|c|c|c|c|c|c|c|}
\hline & \multicolumn{4}{|c|}{ H6 } & \multicolumn{4}{c|}{ H7 } & \multicolumn{4}{c|}{ H8 } & \multicolumn{4}{c|}{ H9 } & \multicolumn{3}{c|}{ H10 } \\
\hline & \multicolumn{2}{|c|}{ h1 } & \multicolumn{2}{c|}{ h2 } & \multicolumn{2}{c|}{ h1 } & \multicolumn{2}{c|}{ h2 } & \multicolumn{2}{c|}{ h1 } & \multicolumn{2}{c|}{ h2 } & \multicolumn{2}{c|}{ h1 } & \multicolumn{2}{c|}{ h2 } & \multicolumn{2}{c|}{ h1 } & \multicolumn{2}{c|}{ h2 } \\
\hline & him & his & hem & hes & him & his & hem & hes & him & his & hem & hes & him & his & hem & hes & him & his & hem & hes \\
\hline $\mathrm{P}_{4}$ & I & & & & & & II & & & & & & & & & & V & & & \\
\hline $\mathrm{P}_{5}$ & & & & & & & & & & & & & & & & I & & & \\
\hline $\mathrm{P}_{6}$ & & & & & & & & & & & & & & & & & & & & \\
\hline
\end{tabular}

\begin{tabular}{|l|c|c|c|c|c|c|c|c|c|c|c|c|c|c|c|c|c|c|c|c|c|c|}
\hline & \multicolumn{4}{|c|}{ H11 } & \multicolumn{4}{c|}{ H12 } & \multicolumn{4}{c|}{ H13 } & \multicolumn{4}{c|}{ H14 } & \multicolumn{3}{c|}{ h15 } \\
\hline & \multicolumn{2}{|c|}{ h1 } & h2 & & \multicolumn{2}{c|}{ h1 } & \multicolumn{2}{c|}{ h2 } & \multicolumn{2}{c|}{ h1 } & \multicolumn{2}{c|}{ h2 } & \multicolumn{3}{c|}{ h2 } & \multicolumn{2}{c|}{ h1 } & \multicolumn{2}{c|}{ h2 } \\
\hline & him & his & hem & hes & him & his & hem & hes & him & his & hem & hes & him & his & hem & hes & him & his & hem & hes \\
\hline $\mathrm{P}_{4}$ & VI & & & & VI & & & & VII & & & & & & & & X & & & \\
\hline$P_{5}$ & II & & II & & III & III & III & & IIII & IIII & IIII & & & V & V & & VI & VI & VI & VI \\
\hline$P_{6}$ & & & & & & & & & & & & & & & & & XI & & & \\
\hline
\end{tabular}

\begin{tabular}{|c|c|c|c|c|c|c|c|c|c|c|c|c|c|c|c|c|c|}
\hline & \multicolumn{4}{|c|}{ H16 } & \multicolumn{4}{c|}{ H17 } & \multicolumn{4}{c|}{ H18 } & \multicolumn{3}{c|}{ H19 } \\
\hline & \multicolumn{2}{|c|}{ h1 } & \multicolumn{2}{c|}{ h2 } & \multicolumn{2}{c|}{ h1 } & \multicolumn{2}{c|}{ h2 } & \multicolumn{2}{c|}{ h1 } & \multicolumn{2}{c|}{ h2 } & \multicolumn{3}{c|}{ h1 } & \multicolumn{2}{c|}{ h2 } \\
\hline & him & his & hem & hes & him & his & hem & hes & him & his & hem & hes & him & his & hem & hes \\
\hline $\mathrm{P}_{4}$ & XI & & & & & & & & I & & & & & & & \\
\hline $\mathrm{P}_{5}$ & & VII & VI & VII & & & X & III & & & & X & & & X & XI \\
\hline$P_{6}$ & & & & & & & & & & & & & & & & \\
\hline
\end{tabular}

tiene en cuenta que la señal en las zonas más alejadas del punto de adquisición puede ser más convulsa. Esto implica que hay más posibilidad de reflexiones múltiples generadas por las partes laterales, debido a las formas complejas de los pilares.

Los datos del georadar permiten identificar tres tramos principales en los fustes de los pilares. El primer tramo, situado en la base del fuste, revela una zona homogénea y de altura variable. El segundo tramo llegará hasta el arranque de las bóvedas, y corresponde a una zona con una composición interna heterogénea e irregular, con anomalías que pueden deberse a cavidades o zonas amorteradas, y también a material no compactado. El último tramo está situado sobre la imposta de la bóveda, y aparece nuevamente una composición interna más homogénea, concentrada en la bóveda.

\section{DISCUSIÓN DE LOS RESULTADOS}

A partir de los datos geométricos obtenidos se observa que la sección de los pilares $\left(\mathrm{P}_{2} \ldots \mathrm{P}_{9}\right)$ y las pilastras $\left(\mathrm{P}_{1}, \mathrm{P}_{10}\right)$ no presenta diferencias significativas de superficie, tanto en la basa como en el fuste. La sección $\left(\mathrm{P}_{2}, \mathrm{P}_{9}\right)$ es algo menor 

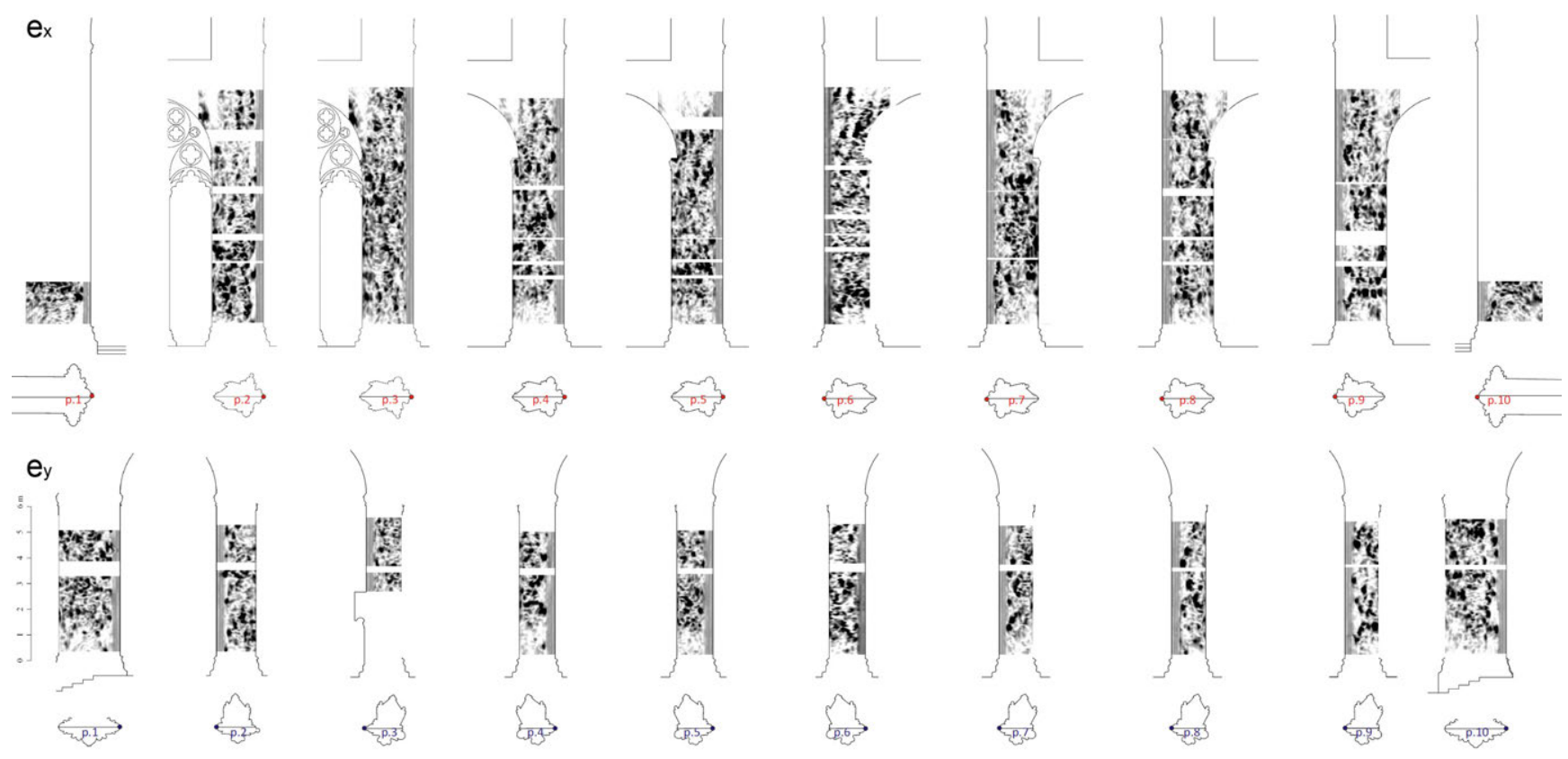

Figura 5. Resultados del georadar para cada pilar. Secciones longitudinal $e_{x} \mathrm{y}$ transversal $e_{y}$. Visualización de energía reflejada.

que la de $\left(\mathrm{P}_{3} \ldots \mathrm{P}_{8}\right)$, aunque éstos han de soportar más carga que los seis restantes del cuerpo central del ábside. Pero lo importante es que la geometría de los pilares $\left(\mathrm{P}_{2} \ldots \mathrm{P}_{9}\right)$ recoge los empujes de las bóvedas que posteriormente han de construirse. El cálculo bidimensional de la sección de la catedral revela un rango de líneas de empujes en un estado límite de la sección (15). La talla y la geometría se realiza en la dirección de su eje principal $e_{x}$. El corte evita los ángulos agudos y las juntas quebradas, la disposición de los lechos y contralechos de la fábrica coincidan con los de la cantera, las juntas son geométricamente planas y el aparejo está bien enlazado. Estos principios son coincidentes con la tradición que perduró hasta las fuentes de la Estereotomía de la Piedra (1897), de Enrique Rovira y Rabasa (1845-1917) (16).

El primer pilar del ábside de la catedral de Tortosa se talla hacia 1377 (ACTo. Ll.o.núm.3, f.66v) que corresponde a la moldura M y a un Maestro A. La disposición general del proyecto de doble deambulatorio debía estar definida. Esta sección $M_{\text {a }}$ se extiende por el muro de cerramiento, hasta la tercera capilla radial. La decisión de eliminar el muro de separación de las capillas absidiales, a diferencia del resto de las cabeceras de la Corona ya estaba tomada. La basa del pilar de la Capilla $\mathrm{C}_{1}$ y su moldura $\mathrm{M}_{\mathrm{b}}$ es diferente a la del resto del ábside, la atribuimos al Maestro B. El resto de las capillas del ábside disponen de una moldura uniforme $\mathrm{M}_{\mathrm{c}}$.

En el inicio de la construcción se observa que la estereotomía y despiece de la bóveda de la capilla $\mathrm{C}_{1}$ (1383), con un mayor número de piezas y juntas de mortero, es diferente a $\mathrm{C}_{2}$ (1387) y las siguientes. La importancia de la tipología de las juntas de mortero aparece en el debate de la catedral de
Gerona (1412) en boca del maestro de Manresa (17), Arnaldo de Valleras ${ }^{4}$. La bóveda puede construirse con un material de menor densidad, mientras que los elementos verticales se han de ejecutar con una piedra de mayor densidad, asignando así la propiedad de dureza de la piedra a aquellos elementos más resistentes. El mortero es para Valleras un elemento importante, ya que un material poroso se agarra mejor para dar continuidad estructural. Todo ello parece determinar la existencia de tres modelos y magisterios diferentes en el inicio de la ejecución del ábside de Tortosa.

En cuanto a la talla de los pilares, éstos disponen de 2 hiladas en la basa y entre 20 y 23 hiladas en el fuste, cuyo rango de alturas está comprendido entre 0,496 m ( $\left.\mathrm{P}_{6}\right)$ y $0,165 \mathrm{~m}\left(\mathrm{P}_{1}\right)$ (Tabla 2). En el inicio de la obra gótica existe un documento del 22 de julio de 1346 en el que se describe el encargo de la talla de 2000 piedras (ACTo.ll.o. 1345-1347, fol.5v-45r). Se indican varios tipos de galgas, referidas a la cana de Tortosa ${ }^{5}$ : $\mathrm{T}_{1}=0,271 \mathrm{~m}, \mathrm{~T}_{2}=0,310 \mathrm{~m}, \mathrm{~T}_{3}=0,349 \mathrm{~m} \mathrm{y} \mathrm{T}_{4}=0,368 \mathrm{~m}$, con un ancho de $0,348 \mathrm{~m}$ y longitud de entre $0,581 \mathrm{~m}$ y $0,697 \mathrm{~m}$ (Figura 6).

El análisis estadístico de las alturas de las hiladas, en relación a las medidas de referencia para el corte de la piedra (Tabla 5), considerando una desviación de hasta \pm 1 dedo $(0,019 \mathrm{~m})$ respecto a cada galga $\left(\mathrm{T}_{1} \ldots \mathrm{T}_{4}\right)$, se establece que, con carácter general, las medidas son coincidentes en un $83,3 \%$. Se observa como la medida más pequeña de $1 \mathrm{p}+2 \mathrm{~d}(0,271 \mathrm{~m})$ es la más utilizada, con un $38,1 \%$. Por el contrario, la altura máxima de $1 \mathrm{p}+2 / 3 \mathrm{p}(0,387 \mathrm{~m})$ se encuentra únicamente en 4 piezas de $\left(\mathrm{P}_{1}\right)$ y $\left(\mathrm{P}_{2}, \mathrm{P}_{2}\right)$. Podemos concluir que las medidas en altura de las hiladas de las basas y de los fustes son coincidentes con los de los rangos establecidos en los libros de fábrica.

\footnotetext{
${ }^{4}$ Es verdad-dice-que la piedra de Manresa es más ligera y amigable para el mortero que la de Gerona y que, si hubiese de construir esta iglesia, haría la bóveda de piedra que fuese más ligera y se pegase mejor el mortero, pero que las crucerías, lo principal, los respaldos y la demás obra, se podrían hacer de piedra de Gerona.

5 El patrón básico de los libros de fábrica es la cana de 8 palmos. La Cana de Tortosa utilizada en la catedral tiene 1.858 cm y el palmo de $23,23 \mathrm{~cm}$.
} 

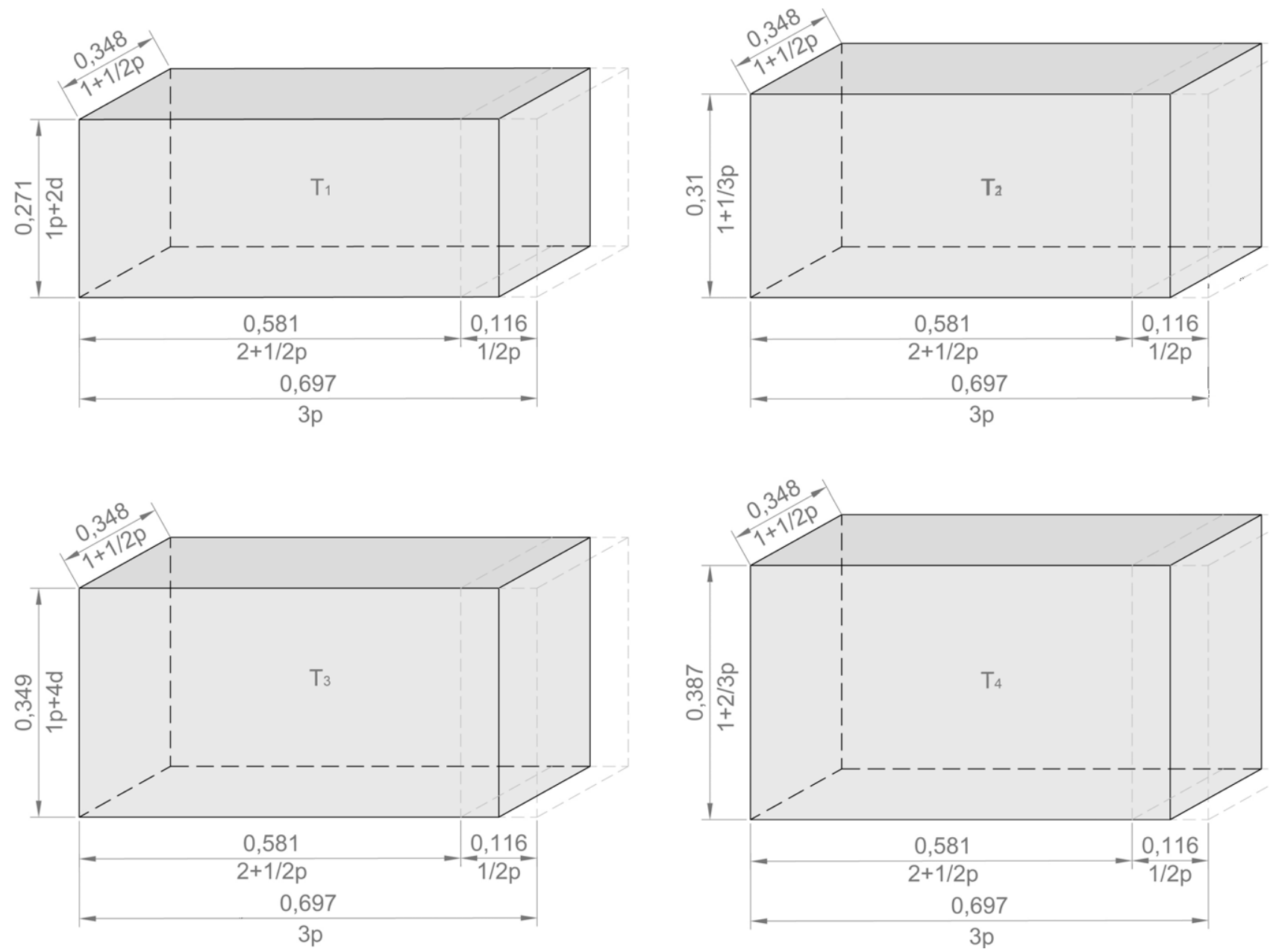

Figura 6. Galgas de referencia según el documento del 22 de julio de 1346 (ACTo.ll.o 1345-1347, fol.5v-45r).

Tabla 5. Análisis estadístico de los cortes de piedra.

\begin{tabular}{|l|c|r|r|r|r|r|r|r|r|r|}
\hline & \multicolumn{2}{|c|}{$\mathbf{0 , 2 7 1}$} & \multicolumn{2}{c|}{$\mathbf{0 , 3 1 0}$} & \multicolumn{2}{c|}{$\mathbf{0 , 3 4 8}$} & \multicolumn{3}{c|}{$\mathbf{0 , 3 8 7}$} & \multicolumn{2}{c|}{ TOTAL } \\
\hline $\mathrm{P}_{1}$ & 5 & $23,8 \%$ & 5 & $23,8 \%$ & 6 & $28,6 \%$ & 1 & $4,8 \%$ & 16 & $76,2 \%$ \\
\hline $\mathrm{P}_{2}$ & 2 & $9,5 \%$ & 6 & $28,6 \%$ & 6 & $28,6 \%$ & 2 & $9,5 \%$ & 14 & $66,7 \%$ \\
\hline $\mathrm{P}_{3}$ & 8 & $38,1 \%$ & 6 & $28,6 \%$ & 4 & $19,0 \%$ & 1 & $4,8 \%$ & 18 & $85,7 \%$ \\
\hline $\mathrm{P}_{4}$ & 11 & $52,4 \%$ & 3 & $14,3 \%$ & 4 & $19,0 \%$ & 0 & $0,0 \%$ & 18 & $85,7 \%$ \\
\hline $\mathrm{P}_{5}$ & 7 & $33,3 \%$ & 9 & $42,9 \%$ & 4 & $19,0 \%$ & 0 & $0,0 \%$ & 20 & $95,2 \%$ \\
\hline $\mathrm{P}_{6}$ & 8 & $38,1 \%$ & 9 & $42,9 \%$ & 0 & $0,0 \%$ & 0 & $0,0 \%$ & 17 & $81,0 \%$ \\
\hline $\mathrm{P}_{7}$ & 12 & $57,1 \%$ & 2 & $9,5 \%$ & 2 & $9,5 \%$ & 0 & $0,0 \%$ & 16 & $76,2 \%$ \\
\hline $\mathrm{P}_{8}$ & 13 & $61,9 \%$ & 3 & $14,3 \%$ & 2 & $9,5 \%$ & 0 & $0,0 \%$ & 18 & $85,7 \%$ \\
\hline $\mathrm{P}_{9}$ & 8 & $38,1 \%$ & 4 & $19,0 \%$ & 1 & $4,8 \%$ & 0 & $0,0 \%$ & 13 & $61,9 \%$ \\
\hline $\mathrm{P}_{10}$ & 11 & $52,4 \%$ & 8 & $38,1 \%$ & 1 & $4,8 \%$ & 0 & $0,0 \%$ & 20 & $95,2 \%$ \\
\hline Med. & 8 & $38,1 \%$ & 6 & $26,2 \%$ & 3 & $14,3 \%$ & - & $0,0 \%$ & 18 & $83,3 \%$ \\
\hline
\end{tabular}

Por otra parte, los pilares $\left(\mathrm{P}_{4}, \mathrm{P}_{5}, \mathrm{P}_{6}\right)$ situados en la parte central del ábside son los que tienen un número mayor de piezas en el fuste con 125, 122 y 165, respectivamente, y son los que disponen de numeración romana. El aumento de piezas tiene la necesidad de ordenar su posterior colocación en la obra. El sistema constructivo gótico tiene una base de prefabricación, pero ésta ha de hacer frente a una necesidad, la colocación del despiece en la fábrica, pasando de 94 piezas en el pilar $\mathrm{P}_{2}$ a 165 en el $\mathrm{P}_{6}$.
La proporción de los cortes de la piedra de los libros de fábrica tendrían la consideración de piedra blanda ${ }^{6}$, según Jean-Baptiste Rondellet (1743-1829) (18). Hay que tener en cuenta que para el maestro gótico el dimensionado de los elementos se realiza en función de la geometría y su dureza (19), como se indica en el tratado Unterweisung (1561) (20), de Lorenz Lechler (c. 1465-c. 1538)7. Todo parece indicar que a mayor densidad, mayor dureza y mayor resistencia, como indican los maestros italianos respondiendo a

\footnotetext{
${ }^{6}$ Corte de piedras, alto, ancho, largo: blanda $(1 ; 1,5 ; 2)$, dureza media $(1 ; 1,5-2 ; 2-3)$, y las duras $(1 ; 2-3 ; 4-5)$.
} 
las objeciones de Jean Mignot ${ }^{8}$ en el debate de la catedral de Milán (1400) (21). La utilización de dos tipos de piedra es frecuente en esta época, el propio Guillem de Sagrera (c. 1380-1454) utiliza una piedra local en la Sala de los Barones de Castel Novo en Nápoles (1453), y otra para los elementos más resistentes, la piedra de Santanyí trasladada desde Mallorca (22).

Desde el punto de vista de la composición interna de los pilares, los resultados obtenidos permiten definir su morfología de forma general. Así, las secciones longitudinales $e_{x} \mathrm{y}$ transversales $e_{y}$ realizadas permiten deducir que los pilares son fundamentalmente macizos, tanto en la base como en las tres primeras hiladas del fuste (Tabla 6). Complementariamente, los resultados del georadar permiten identificar en algunos pilares zonas homogéneas relevantes en cotas superiores. Es posible discretizar así los pilares según su composición interna: $\left(\mathrm{P}_{2}, \mathrm{P}_{3}\right),\left(\mathrm{P}_{4}, \mathrm{P}_{5}\right),\left(\mathrm{P}_{6}, \mathrm{P}_{7}, \mathrm{P}_{9}\right)$. Los pilares $\left(\mathrm{P}_{2}, \mathrm{P}_{3}\right)$ presentan una estructura interna similar, en la que se identifican zonas con bloques masivos con un espesor de $0,3 \mathrm{~m}$. Cabe mencionar también la singularidad del pilar $\left(\mathrm{P}_{8}\right)$, con tres tramos homogéneos, tal y como se identifica en los pilares $\left(\mathrm{P}_{2}\right.$ y $\left.\mathrm{P}_{3}\right)$, aunque en este caso sí es posible determinar las alturas de los tramos (Tabla 7).

\section{CONCLUSIONES}

En la catedral de Tortosa se establece un nuevo modelo del gótico meridional con la construcción de un doble deambulatorio, al suprimir el muro entre las capillas radiales. Éstas tienen una proporción muy baja de $9 / 5$, en relación a otros modelos, y el diseño del pilar es un elemento esencial, ya que concentra la potencia de la estabilidad global de la fábrica. La forma alargada según su sección principal $e_{x}$ garantiza esta primera fase constructiva, pero además el pilar está preparado para la transformación funcional que representará la construcción de la girola.

En la construcción de las capillas radiales se determinan cinco momentos constructivos según la morfología de los pilares (Figura 7). El primero (1377-1378) supone la construcción de la pilastra $\mathrm{P}_{1}$ y parte cerramiento exterior con la moldura $\mathrm{M}_{\mathrm{a}}$. El modelo de doble deambulatorio se definió aquí, y el despiece de la cantería tiende a tener muy pocas juntas. En un segundo momento constructivo (1378-1383) se construye el $\mathrm{P}_{2}$, con una moldura $\mathrm{M}_{\mathrm{b}}$, y la bóveda de la Capilla $\mathrm{C}_{1}$. El pilar $\mathrm{P}_{2}$ es el que tiene menos juntas de todos los del deambulatorio, y además el corte de las piedras de la bóveda es más pequeño que en el resto $\left(\mathrm{C}_{2} \ldots \mathrm{C}_{9}\right)$. En este momento se comprueba la estabilidad del nuevo modelo. El tercer momento (1383-1388) implica la construcción del pilar $\mathrm{P}_{3}$, con una base $\mathrm{M}_{\mathrm{c}}$. El despiece del pilar aumenta considerablemente respecto a $\mathrm{P}_{2}$, de 94 piezas se pasa a 126 , con lo que disminuye el grado de exigencia de la talla, reduciendo considerablemente

Tabla 7. Morfología interna de los pilares y alturas (a) de los tramos homogéneos identificados.

\begin{tabular}{|c|c|c|c|c|}
\hline & & hg. long. & hg. trans. & Composición \\
\hline \multirow{2}{*}{$\mathrm{P}_{1}$} & a1 & $0-1,8$ & $0-1,8$ & $0,7(0,3+0,4)$ \\
\hline & a2 & n.d. & $1,8-5,5$ & 0,3 \\
\hline \multirow{3}{*}{$\mathrm{P}_{2}$} & a1 & $0-2,1$ & $0-2,1$ & $1,2(0,3+0,4+0,5)$ \\
\hline & a2 & $2,1-6,1$ & 2,1-n.d. & 0,3 \\
\hline & a3 & $6,1-10$ & n.d. & n.d. \\
\hline \multirow{3}{*}{$\mathrm{P}_{3}$} & a1 & $0-2,1$ & n.d. & $1,2(0,3+0,4+0,5)$ \\
\hline & a2 & $2,1-7,2$ & n.d. & 0,3 \\
\hline & a3 & $7,2-10$ & n.d. & n.d. \\
\hline \multirow{2}{*}{$\mathrm{P}_{4}$} & a1 & $0-1,8$ & $0-1,8$ & homogéneo \\
\hline & a2 & $1,8-2,4$ & $1,8-2,4$ & 0,6 \\
\hline \multirow{2}{*}{$\mathrm{P}_{5}$} & a1 & $0-1,5$ & $0-1,5$ & homogéneo \\
\hline & a2 & $1,5-2,4$ & $1,5-2,4$ & 0,65 \\
\hline$P_{6}$ & a1 & $0-1,3$ & $0-1,3$ & homogéneo \\
\hline $\mathrm{P}_{7}$ & a1 & $0-1,5$ & $0-1,5$ & homogéneo \\
\hline \multirow{3}{*}{$\mathrm{P}_{8}$} & a1 & $0-1,5$ & $0-1,5$ & homogéneo \\
\hline & a2 & $1,5^{-1,9}$ & $1,5-1,9$ & 0,8 \\
\hline & a3 & $1,9-2,4$ & $1,9-2,4$ & 0,55 \\
\hline $\mathrm{P}_{9}$ & a1 & $0-1,6$ & $0-1,6$ & homogéneo \\
\hline $\mathrm{P}_{10}$ & a1 & n.d. & $0-1,8$ & $1,4(0,3+0,45+0,75)$ \\
\hline
\end{tabular}

Tabla 6. Estructura interna en el arranque de los pilares. Altura ( $h$ y número de piezas ( $n$ ) para cada hilada (B, H). Altura homogénea en la base según el georadar $(h g)$.

\begin{tabular}{|c|c|c|c|c|c|c|c|c|c|c|c|c|c|}
\hline & & & & & B & & $\mathbf{H}$ & & H & & $\mathbf{H}$ & & \\
\hline & hg. long & hg. tran. & $\boldsymbol{h}$ & $n$ & $\boldsymbol{h}$ & $n$ & $\boldsymbol{h}$ & $n$ & $\boldsymbol{h}$ & $n$ & $\boldsymbol{h}$ & $n$ & Total \\
\hline$P_{1}$ & 1,800 & 1,800 & 0,343 & 5 & 0,486 & 2 & 0,371 & 2 & 0,278 & 2 & 0,349 & 2 & 1,826 \\
\hline $\mathrm{P}_{2}$ & n.d. & & 0,343 & 2 & 0,443 & 6 & & & & & & & \\
\hline $\mathrm{P}_{3}$ & n.d. & & 0,356 & 7 & 0,422 & 8 & & & & & & & \\
\hline $\mathrm{P}_{4}$ & 1,800 & 1,800 & 0,339 & 5 & 0,437 & 6 & 0,336 & 6 & 0,330 & 7 & 0,257 & 6 & 1,700 \\
\hline $\mathrm{P}_{5}$ & 1,500 & 1,600 & 0,346 & 12 & 0,433 & 11 & 0,273 & 5 & 0,291 & 4 & 0,287 & 6 & 1,631 \\
\hline $\mathrm{P}_{6}$ & 1,300 & 1,350 & 0,257 & 12 & 0,496 & 11 & 0,312 & 6 & 0,300 & 4 & & 6 & 1,365 \\
\hline $\mathrm{P}_{7}$ & 1,500 & $1,55^{\circ}$ & 0,339 & 6 & 0,428 & 6 & 0,288 & 7 & 0,252 & 6 & 0,260 & 6 & 1,567 \\
\hline $\mathrm{P}_{8}$ & 1,500 & 1,400 & 0,348 & 7 & 0,414 & 6 & 0,312 & 6 & 0,250 & 4 & 0,227 & 5 & 1,551 \\
\hline $\mathrm{P}_{9}$ & 1,600 & 1,600 & 0,329 & 4 & 0,434 & 4 & 0,284 & 7 & 0,286 & 3 & 0,290 & 6 & 1,623 \\
\hline$P_{10}$ & n.d. & 1,800 & 0,324 & 5 & 0,428 & 4 & 0,314 & 2 & 0,317 & 2 & 0,326 & 2 & 1,708 \\
\hline
\end{tabular}

\footnotetext{
7 hay que saber si la piedra es dura o blanda... si la piedra es buena, quita tres pulgadas al espesor del muro, si fuese mala aumenta tres pulgadas.

8 Los contrafuertes de dicha iglesia son lo suficientemente fuertes y capaces de sostener su peso, pero por muchas más razones, ya que un brazo de nuestro mármol y nuestro sarrizio, cualquiera que sea su anchura, es tan resistente como dos brazos de las piedras francesas...
} 


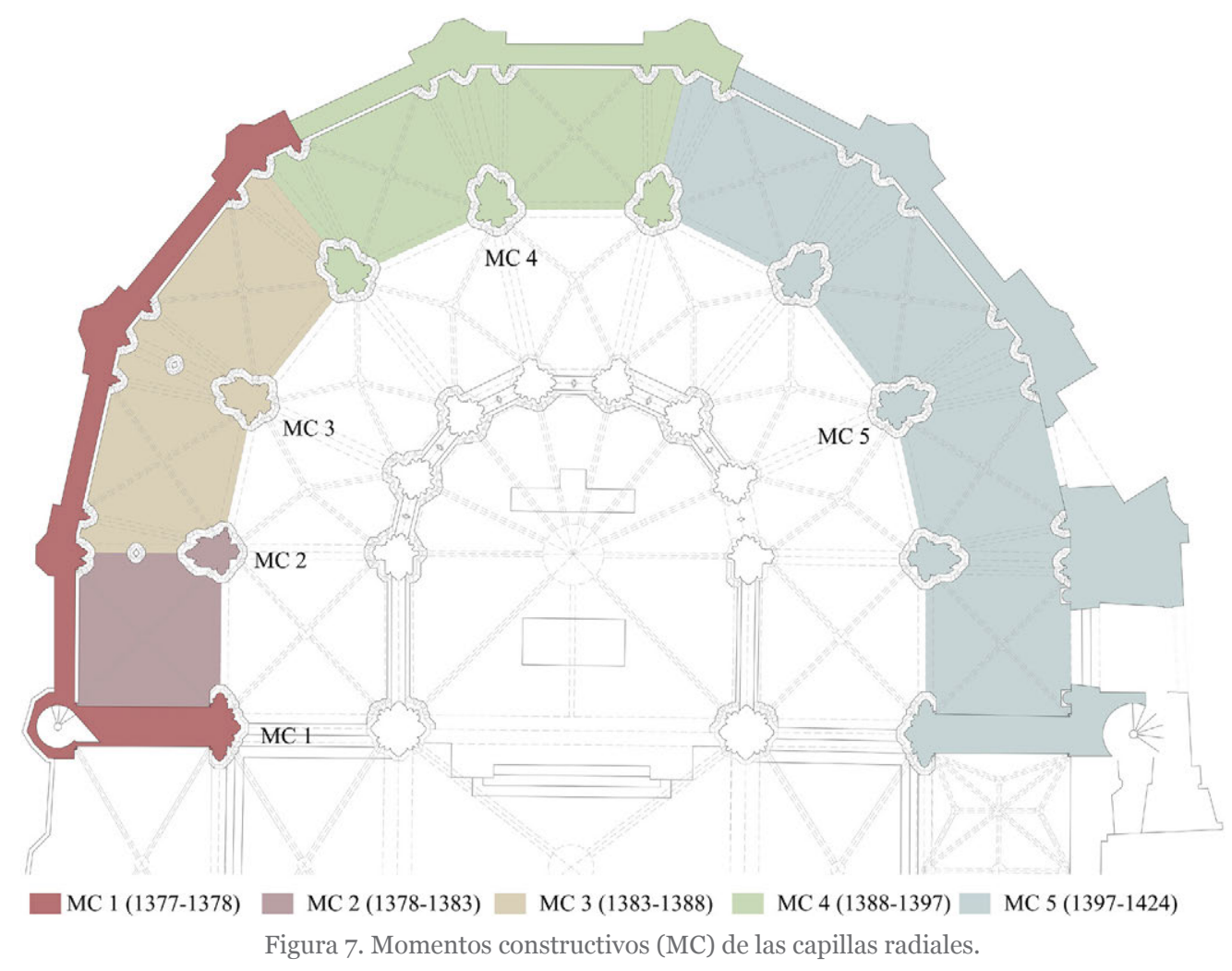

el peso de las piezas. Se establecería así la consolidación del modelo con el cierre de la capilla $\left(\mathrm{C}_{2}\right)$. El cuarto momento (1388-1397) representará la construcción de los pilares $\left(\mathrm{P}_{4}\right.$, $\mathrm{P}_{5}, \mathrm{P}_{6}$ ). Éstos son los pilares con un mayor número de piezas y por ello han de ser numeradas mediante números romanos. El último tramo (1397-1424) morfológicamente supone la quinta fase constructiva, con los pilares $\left(\mathrm{P}_{7}, \mathrm{P}_{8}, \mathrm{P}_{0}\right)$ y la pilastra $\mathrm{P}_{10}$, y se caracteriza por una disminución del número de piezas que componen los pilares respecto a la fase anterior. La posterior construcción de la girola, ejecutada por la misma familia de maestros de obra (1432-1434), provocó un aumento en la altura de la sección, con una proporción de 9/6. El aumento de la seguridad en el despiece de los pilares podría ser la respuesta de volver a disminuir el número de las piezas, pasando de las 165 piezas en $\mathrm{P}_{6}$ a las 108 del $\mathrm{P}_{9}$. Por otra parte, la homogeneidad del interior de los pilares en su arranque sí es constante en las cinco tipologías determina-

das, y en todas ellas el fuste empieza a construirse con tres hiladas también macizas.

La tradición de la talla de la piedra indica la importancia de la relación entre el material, el contacto entre elementos y la disposición del corte, de acuerdo con la ley de la piedra. En términos de estereotomía, la piedra utilizada en la catedral de Tortosa es blanda. Por tanto, hay que añadir otros factores de seguridad para garantizar la estabilidad del nuevo modelo. En primer lugar, una sección geométrica muy baja de $9 / 5$. Otro es el derivado del material y la gran medida de las piezas en el inicio del modelo. Sólo así puede justificarse la utilización de estos grandes prefabricados, en la confianza de garantizar la estabilidad de un modelo no experimentado. En términos de práctica del maestro medieval, a menos juntas, mayor homogeneidad de material, mayor consistencia y, por tanto, mayor resistencia.

\section{REFERENCIAS}

(1) Alonso, M. (1955). De Scientiis. Compilación a base principalmente de la de Al-Farabi, Apéndice I. Madrid: Consejo Superior de Investigaciones Científicas.

(2) Lluis i Ginovart, J. (2009). Evolución constructiva de los pilares de una girola gótica. El concepto de homogeneidad del material versus resistencia. En: Actas del Sexto Congreso Nacional Historia de la Construcción, pp. 733-743. Madrid: Instituto Juan de Herrera.

(3) Núñez, A., Buill, F., Regot, J., de Mesa, A. (2012). Generation of virtual models of cultural heritage. Journal of Cultural Heritage, 13: 103-106.

(4) Armesto, J., Roca-Pardiñas, J., Lorenzo, H., Arias, P. (2010). Modelling masonry arches shape using terrestrial laser scanning data and nonparametric methods. Engineering Structures, 32: 607-615.

(5) Alkan, R.M., Karsidag, G. (2012). Analysis of the Accuracy of Terrestrial Laser Scanning. Measurements (May 2012): 6-10.

(6) Guarnieri, A., Vettore, A., Remondino, F. (2004). Photogrammetry and ground-based laser scanning: assessment of metric accuracy of the 3D model of Pozzoveggiani Church. En Working Week, The Olympic Spirit in Surveying. Athens. 
(7) Grussenmeyer, P., Landes, T., Voegtle, T., Ringle, K. (2008). Comparison methods of terrestrial laser scanning, photogrammetry and tacheometry data for recording of cultural heritage buildings. International Archives of Photogrammetry, Remote Sensing and Spatial Information Sciences, 37 (B5): 213-218.

(8) Dabas, M., Camerlynck, C., Freixas i Camps, P. (2000). Case History: Simultaneous use of electrostatic quadrupole and GPR in urban context: Investigation of the basement of the Cathedral de Girona (Catalunya, Spain). Geophysics, 65(2): 526-532.

(9) Pérez, V., Canas, J.A., Pujades, Ll., Clapés, J., Caselles, O., García, F., Osorio, R. (200o). GPR survey to confirm the location of ancient structures under the Valencian Cathedral (Spain). Journal of Applied Geophysics, 43: 167-174.

(10) Vendrell, M., Giráldez, P., González, R., Cavallé, F., Roca, P. (2008). Santa Maria del Mar. Estudi històric-constructiu, materials de construcció i estabilitat estructural. Barcelona: UB-Patrimoni, Veclus, UPC.

(11) González, J., Roca, P. (2004 y 2008). Estudio, diagnóstico y peritación y en su caso planteamiento de actuaciones sobre el comportamiento de la catedral de Santa María, en la ciudad de Palma, isla de Mallorca (Baleares) Fase I y Fase II. Barcelona: Universitat Politècnica de Catalunya, por encargo del Ministerio de Cultura.

(12) Valentini, G. (1990). Il Duomo di Milano. Una disputa medievale sul modello del tempio, pp. 61-78. Milán: Nuovo Edizioni Duomo.

(13) Viollet-le-duc, E. (1854-1868). Dictionnaire raisonné de l'architecture française du xie. au xvie. siècle, vol. 4, pp. 167173. Paris: Libraires-Imorimiers Réunies.

(14) Kimpel, D. (1980). L'apparition des elements de serie dans les grands ouvrages. Historie et Archeologique. Les batisseurs de Moyen-Age, n. ${ }^{\circ} 47$, novembre, pp. 40-59.

(15) Lluis i Ginovart, J., Costa-Jover, A., Fortuny i Anguera, G. (2015). Elementos auxiliares de construcción en la arquitectura gótica. El pilar «major» de la catedral de Tortosa. Informes de la Construcción, 67(537): e057, doi: http://dx.doi. org/10.3989/ic.13.059.

(16) Rovira, E. (1897). Estereotomía de la Piedra, pp. 220-221. Barcelona: Librería y Estampería Artística.

(17) Llaguno, E., Ceán, J.A. (1829). Noticia de los Arquitectos y Arquitectura de España desde su restauración, pp. $261-275$. Madrid: Imprenta Real.

(18) Rondelet, J.B. (1804). Traité thèorique et pratique de l'Art de Bâtir par Jean Rondelet, tome second, pp. 20-22. Paris: Chez l'auteur, enclos du Panteón, Les dimensions des pierres. Plancha n. ${ }^{\circ}$ XVII.

(19) Huerta, S. (2007). Las reglas estructurales del gótico tardío alemán. En Actas del Quinto Congreso Nacional de la Construcción, pp. 519-532. Madrid: Instituto Juan de Herrera.

(20) Ruiz de la Rosa, J.A. (1987). Traza y Simetría de la Arquitectura en la antigüedad y Medievo, pp. 326-329. Sevilla: Servicio de Publicaciones de la Universidad de Sevilla.

(21) Yarza, J., et. al. (1982). Arte Medieval II. Románico y Gótico, pp. 329-337. Barcelona: Gustavo Gili.

(22) Serra, A. (2000). «È cosa catalana» la Gran Sala de Castel Nuovo en el contexto mediterráneo. Annali di architettura. Rivista del Centro internazionale de Studi di Architettura Andrea Palladio di Vicenza, n. ${ }^{\circ}$ 12: 7-16. 